\title{
Bursting detection by Variable-Interval Time-Averaging (VITA) on spanwise oscillated turbulent channel flow
}

\author{
Atilla Altıntaş \\ Karabuk University, Engineering Faculty, 78050 Karabuk, Turkey
}

\begin{abstract}
The main aim of the study is to detect the bursting phenomenon in spanwise oscillated Lorentz force applied turbulent channel flow. Regarding the biggest reason of the turbulence production is known as the bursting phenomenon, detecting the decrease on it could be a very effective tool as an estimation of the effectiveness of the applied control strategy to the flow. The data gained by DNS (Direct numerical simulations) at low Reynolds number $\left(R e_{\tau}=180\right.$ based on the wall-shear velocity and channel half width). The VariableInterval Time-Averaging (VITA) technique has been applied to three different streamwise locations for both applied force and no-force cases. The Lorentz force applied only to the lower wall. In first step the very well known drag reduction is observed, we compared the drag reduction results for the applied force and no-force cases both for the lower and upper wall of the channel. The bursting detection results are given statistically, which clearly shows that bursting phenomenon frequency tend to decrease as a result of the applied Lorentz force. It is also has shown that associated turbulence production has similar trend.
\end{abstract}

\section{Introduction}

The flow control studies are attracting a great attention since Prandtl successfully controlled a boundary layer [1], many numerical, experimental and theoritical studies are performed to control the drag in turbulent boundary layers [2-7]. One of the effective methods of the drag control is known as Lorentz force excitation. In this method, the magnets and electrodes are placed on the wall and by choosing an electrically conducting fluid the Lorentz force is generated in the desired direction.

The Lorentz force applications are performed in all three directions (streamwise, spanwise and wall-normal). It seems better results are obtained for spanwise direction [6].

It is known that Lorentz force used by [2] for flow control for the first time. They applied streamwise Lorentz force to a laminar boundary layer in order to delay the transition to turbulence of the flow over submerged vehicles. A more stable Blasius boundary layer profile under the influence of the Lorentz force is obtained by [8]. Later some experimental studies performed which explored the possibility of achieving viscous drag reduction in turbulent boundary layers [9]. An another important experimental study was later performed by [4], they placed the magnets and electrodes to direct the electromagnetic (EM) body force axially downstream and axially upstream. In these experiments a turbulence suppression up to $30 \%$ was achieved for the former case with the interaction parameter $(S t \sim O(1)$ is used, which represents the so-called "interaction parameter" is the ratio of the Lorentz force to the inertial force. However, with the body force axially upstream these researchers found an augmentation of the turbulence.

O'Sullivan and Biringen showed that feedback control schemes are capable to reduce skin friction in turbulent boundary layers [10].

A DNS study of a turbulent channel flow performed at different low Reynolds numbers $\left(R e_{\tau}=100,200,400\right)$, in which an idealized Lorentz force is applied for both open-loop and closed-loop configurations. In an idealized open-loop case they achieved up to $40 \%$ drag reduction. They provided a relation between $S t$ number and a given Reynolds number for the best drag reduction [6].

This paper presents DNS simulations of channel flow (Reynolds number of 180) in which spanwise oscillating Lorentz force is applied along the spanwise direction. The aim of the paper is to figure out the bursting frequency under the influence of the spanwise oscillated Lorentz force in turbulent channel flow.

The paper is organized as follows. First, the methodology and equations are presented followed by a brief description of the numerical method. In the next section the results are presented and discussed and then some conclusing remarks are given.

\section{Methodology}

\subsection{Governing equations}

The resulting force can be estimated as a body force and directly added as a body force term to the Navier-Stokes 
equations. Thus, the governing equations take the following nondimensional form

$$
\begin{gathered}
\frac{\partial \mathbf{u}}{\partial t}+\mathbf{u} \cdot \nabla \mathbf{u}=-\nabla \mathbf{p}+\frac{1}{R e_{\tau}} \nabla^{2} \mathbf{u}+S t(\mathbf{J} \times \mathbf{B}), \\
\nabla \cdot \mathbf{u}=0,
\end{gathered}
$$

where $R e_{\tau}$ is the Reynolds number based on $u_{\tau}$ (wall shear velocity) and $\delta$ (half channel height). $S t=J_{0} B_{0} \delta /\left[\rho u_{\tau}^{2}\right]$, is the Stuart number which represents the relative strength of the Lorentz force with respect to the inertia force, where $J_{0}$ and $B_{0}$ are the current density and the magnetic flux density values at the wall, respectively.

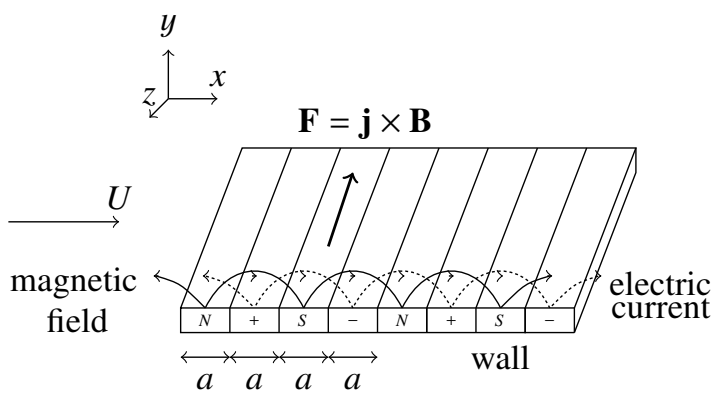

Figure 1. Illustration of magnets and electrodes arrangement for generating a Lorentz force along the spanwise direction [6].

\subsection{Force}

The spanwise force oscillates in time and decays exponentially in the wall-normal direction,

$$
\left.f_{z}^{+}=S t \exp -\frac{\pi y^{+}}{a^{+}}\right) \sin \left(\frac{2 \pi t^{+}}{T^{+}}\right) .
$$

According to [6] the rule defining the effective $S t$ value for different Reynolds numbers given as

$$
\left.\frac{S t T^{+}}{R e_{\tau} \pi}\right)_{o p t}=20 .
$$

We performed simulations for $R e_{\tau}=180$. For $T^{+}=100$, equation 4 gives $S t=36 \pi$. The parameter $T^{+}$is the period of oscillation, and $a^{+}$(the magnet and electrode widths, which here are assumed to be the same) sets the distance the force penetrates into the flow; it is here taken as $a^{+} / \pi=\Delta^{+}=10$. Lorentz force excitation is applied along the spanwise direction for forcing the flow to oscillate at a certain frequency.

The required Lorentz force can be created by placing electrodes and magnets side by side, in the streamwise direction parallel to one another, as shown in figure 1 . This configuration generates Lorentz force in the spanwise direction which decays exponentially in the wall-normal direction. We apply the force in the lower half of the channel, but because of the exponential function it is active only for $y^{+}=60$.

\subsection{Direct numerical simulations}

An incompressible, finite volume code is used [11]. The numerical procedure is based on an implicit, fractional step technique with a multigrid pressure Poisson solver and a non-staggered grid arrangement. For the momentum equations, central differencing is used in space and the Crank-Nicolson scheme is used in the time domain.

A constant volumetric driving force is used in the streamwise momentum equation by which the frictional Reynolds number, $R e_{\tau}=180$, is prescribed. Periodic boundary conditions are used in the streamwise and spanwise directions while the usual no-slip boundary conditions are enforced at the walls. The domain size is $2 \pi \delta \times \pi \delta \times 2 \delta$ in the streamwise, spanwise and wall-normal directions, respectively with grid size $98 \times 98 \times 98$. The superscript ' + ' sign indicates a nondimensional quantity scaled by the wall variables: e.g. $y^{+}=y u_{\tau} / v$, where $v$ is the kinematic viscosity, $u_{\tau}$ is the wall-shear velocity. In this work $u, v, w$ represents the streamwise, wall-normal and spanwise velocities respectively. Before applying any control, all simulations are allowed to reach a fully developed turbulent flow state.

\section{Results and discussion}

In figure 2 the time histories of drag forces in the vicinity of the wall, which is dominated by wall shear stress, $\tau_{w}$, is demonstrated. In figure 2(a) there is not any control, in which the lower and upper wall exhibits similar drag forces. However in figure 2(b) the spanwise oscillated Lorentz force is applied to the lower wall, which exhibits a significant drag reduction compare to upper, no-force applied wall.

Figure 3(b) and figure 3(a) represents the contour plots for the spanwise velocity at the location of half the streamwise length of the channel $(x=\pi)$. Comparison of the both cases suggests that in the applied force case the vortex structures near the lower wall are suppressed in applied force case compared to no-force case.

\subsection{Bursting detection comparison}

It is now very well known that the biggest reason of the turbulence production is the bursting phenomenon which accounts approximately $70 \%$ of the turbulence production [12-14]. Here we applied the Variable-Interval TimeAveraging, (VITA) technique to detect the bursting frequency in both the force applied and no-force cases. For a fluctuating quantity $Q\left(x_{i}, t\right)$ the variable-interval time average is defined by [15]

$$
\widehat{Q}\left(x_{i}, t, T\right)=\frac{1}{T} \int_{t-\frac{1}{2} T}^{t+\frac{1}{2} T} Q\left(x_{i}, \tau\right) \mathrm{d} \tau,
$$

here $T$ is the averaging time. As $T$ becomes large, approaches to conventional averaging results. To represent a measure of the turbulent energy during time $T$ a localized variance is defined as;

$$
\widehat{\operatorname{var}}\left(x_{i}, t, T\right)=\widehat{u^{\prime 2}}\left(x_{i}, t, T\right)-\left[\widehat{u^{\prime}}\left(x_{i}, t, T\right)\right]^{2},
$$




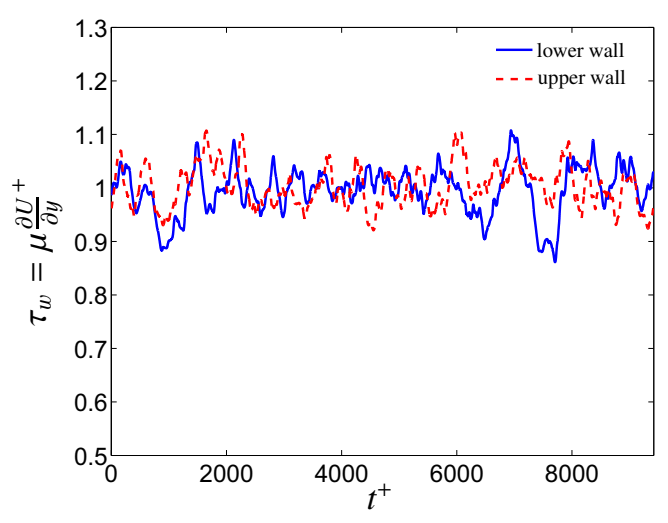

(a) No-force applied case.

Figure 2. Normalized drag history, $\operatorname{Re}_{\tau}=180$.

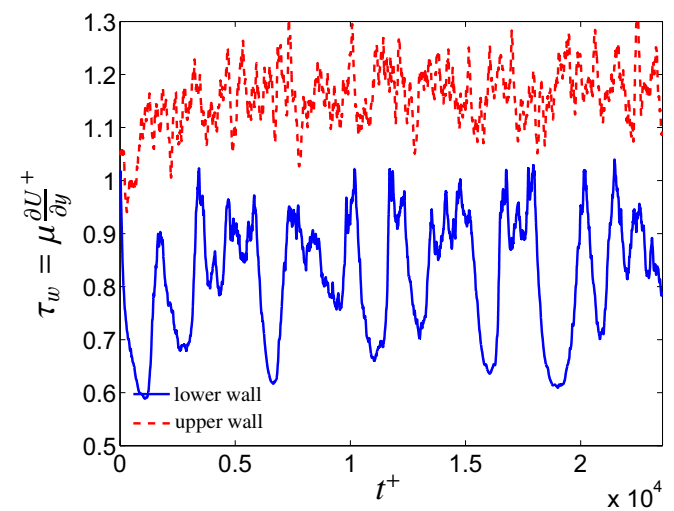

(b) Force applied case.

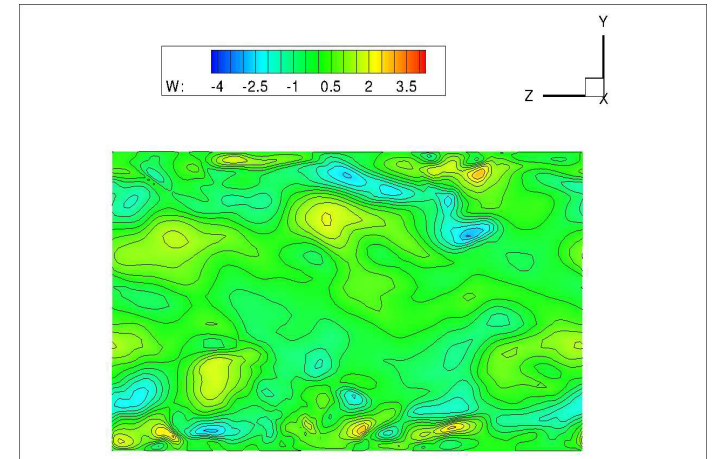

(a) No force applied case.

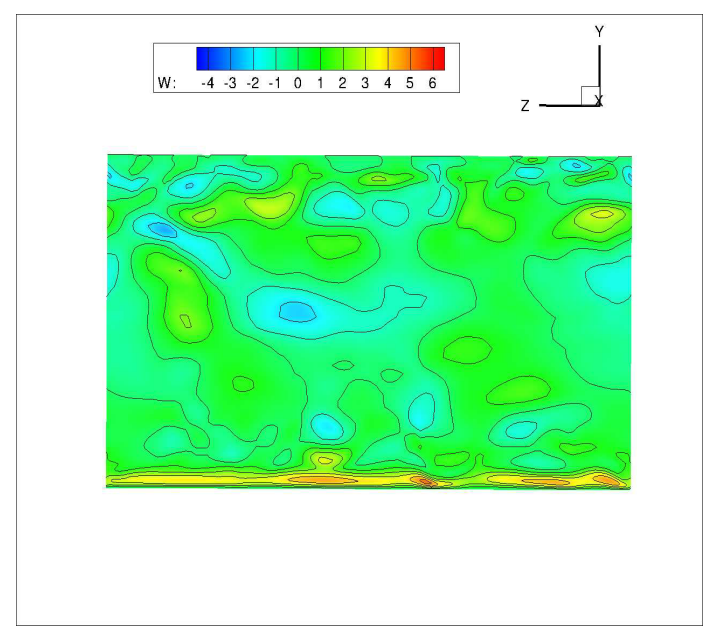

(b) Force applied case

Figure 3. Spanwise velocity field, contour plots from $x=\pi . R e_{\tau}=180$.

The detection function $D(t)$ is defined as;

$$
D(t)= \begin{cases}1, & \text { for } \widehat{\operatorname{var}}>k u_{r m s}^{\prime 2} \text { and } \frac{\partial u^{\prime}}{\partial t}>0 \\ 0, & \text { otherwise }\end{cases}
$$

where $k$ is the threshold level, which here taken as $k=1$ for detecting strongest structures responsible for the turbulence production [16]. The result of the bursting detections and related turbulence production are figured out in figure 4. The turbulence production values are compared with DNS data [17]. The data has been gathered at four different streamwise locations and at 10 different wall-normal locations for each streamwise locations. The streamwise locations are $x^{+}=275,565$ and 1036. The wall-normal locations are $y^{+}=5,10,15,20,30,40,50,62$.

It is observed that a big amount of the the bursting phenomenon occures at the vicinity of the wall $\left(y^{+}<20\right)$ (figures 4(a),4(b),4(c)). As expected, the turbulence production results also parallel with the bursting frequency results which indicates that the most of the turbulence production occures at the vicinity of the wall (figure 4(d)).

The bursting frequency results clearly indicates that in all three different locations the bursting phenomenon occures in a much less frequency for the applied force cases compare to no-force cases (figures 4(a),4(b),4(c)). Beside the turbulence production values are lower at applied force wall for the applied force case compare to no-force case which is coherent with bursting detection results.

\section{Conclusions}

A DNS study of a fully developed turbulent channel flow is performed where a spanwise oscillating Lorentz force has applied near the lower wall (figure 1). Very well known drag reduction is obtained first (figure 2), then bursting phenomenon as well as related turbulence productions are investigated. The spanwise velocity contours are depicted 


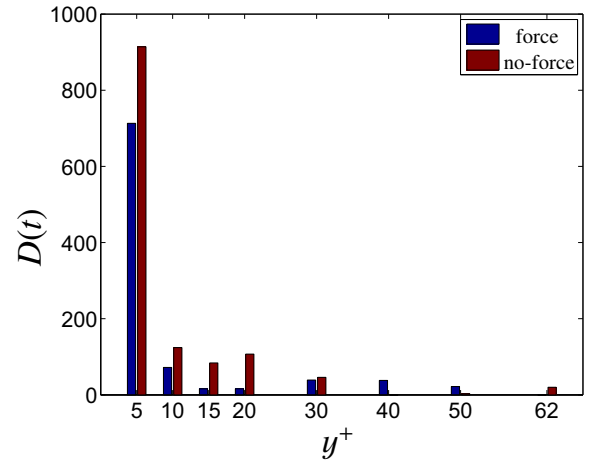

(a) Bursting detection at $x^{+}=275$.

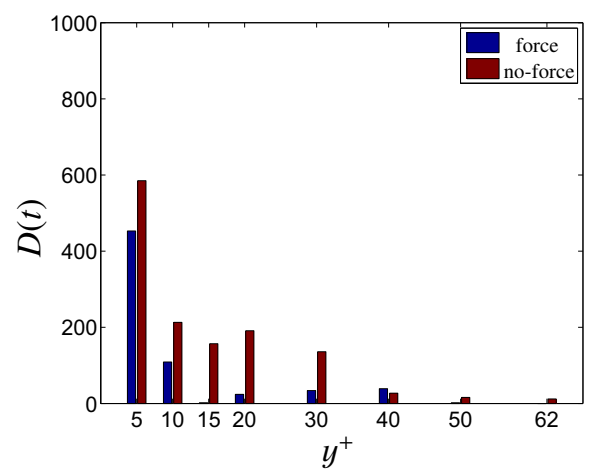

(c) Bursting detection at $x^{+}=1036$.

Figure 4. Burst detection at three different streamwise locations.

which clearly indicates that vorticies are suppressed in applied force case (figure 3). The VITA technique has been used to detect the bursting frequency which investigated at three different streamwise locations for ten different wallnormal locations.

We found that in the applied force cases bursting phenomenon occures in a less frequency compare to no-force cases at all three locations (figures 4(a),4(b),4(c)). We also support these results with related turbulence production values, which indicates that in applied force case turbulence production has lower values compare to no-force case (figure 4(d)).

\section{References}

[1] L. Prandtl, NACA TM 452 (1904)

[2] A. K. Gailitis, O. A. Lielausis, Appl. Magnetohydrodyn. Trudy Inst. 12, 143-146 (1961)

[3] H. Choi, P. Moin, J. Kim, J. Fluid Mech. 261, 75-110 (1994)

[4] C. Hennoch, J. Stace, Phys. Fluids 7, 1371-1383 (1995)

[5] C. H. Crawford, G. E. Karniadakis, Phys. Fluids 9, 788-806 (1997)

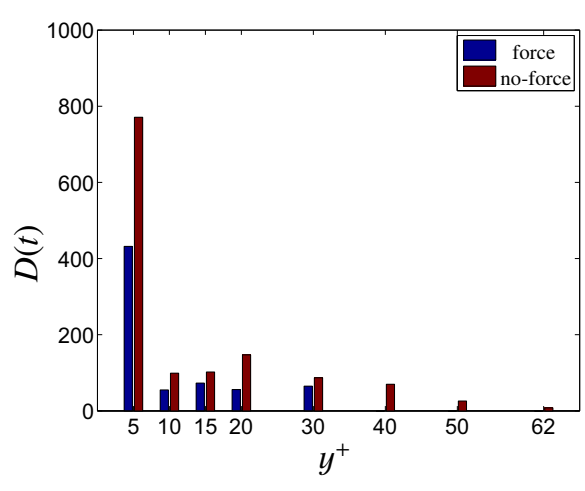

(b) Bursting detection at $x^{+}=565$.

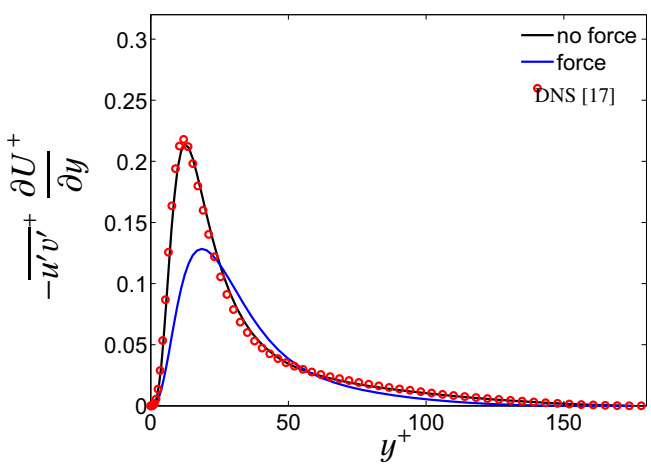

(d) Turbulence production rate, lower wall, $R e_{\tau}=180$.

[6] T. W. Berger, J. Kim, C. Lee, Phys. Fluids 12, 631-649 (2000)

[7] T. Albrecht, H. Metzkes, R. Grundmann, G. Mutschke, G. Gerbeth, Magnetohydrodynamics 44, 205-222 (2008)

[8] A. B. Tsinober, A. G. Shtern, English translation: Magnetohydrodynamics 3, 103-105 (1967)

[9] D. M. Nosenchuck, G. L. Brown, in Near-Wall Turbulent Flows Elsevier Science edited by R. M. C. So, C. G. Speziale, and B. E. Launder, Publishers B.V., New York, 689-698 (1993)

[10] P. L. O’Sullivan, S. Biringen, Phys. Fluids 10, 11691181 (1998)

[11] L. Davidson, S.-H Peng, Int. J. Numer. Fluids 43, 1003-1018 (2003)

[12] H. T. Kim, S. J. Kline, W. C. Reynolds, J. Fluid Mech. 50, 133-160 (1972)

[13] J. Kim, NASA TM 85944 (1984);

[14] J. Kim, Phys. Fluids 28, 52-58 (1985);

[15] R. F. Blackwelder, R. E. Kaplan, J. Fluid Mech. 76, 89-112 (1976)

[16] M. Ichimiya, I. Ikuo Nakamura, S. Yamashita, Exp. Thermal and Fluid Science, 17 37-48 (1998)

[17] R. D. Moser, J. Kim, N. N. Mansour, Phys. Fluids, 11 943-945 (1999) 